Review Article

\title{
Contribution of Neuronal and Glial Two-Pore-Domain Potassium Channels in Health and Neurological Disorders
}

\author{
Yuncheng Luo, Lu Huang, Ping Liao, and Ruotian Jiang $\mathbb{B}$ \\ Laboratory of Anesthesia and Critical Care Medicine, National-Local Joint Engineering Research Center of Translational Medicine \\ of Anesthesiology, West China Hospital, Sichuan University, Chengdu 610000, China
}

Correspondence should be addressed to Ruotian Jiang; ruotianjiang@scu.edu.cn

Received 28 June 2021; Accepted 3 August 2021; Published 15 August 2021

Academic Editor: Fang Pan

Copyright ( 2021 Yuncheng Luo et al. This is an open access article distributed under the Creative Commons Attribution License, which permits unrestricted use, distribution, and reproduction in any medium, provided the original work is properly cited.

\begin{abstract}
Two-pore-domain potassium (K2P) channels are widespread in the nervous system and play a critical role in maintaining membrane potential in neurons and glia. They have been implicated in many stress-relevant neurological disorders, including pain, sleep disorder, epilepsy, ischemia, and depression. K2P channels give rise to leaky $\mathrm{K}^{+}$currents, which stabilize cellular membrane potential and regulate cellular excitability. A range of natural and chemical effectors, including temperature, pressure, $\mathrm{pH}$, phospholipids, and intracellular signaling molecules, substantially modulate the activity of K2P channels. In this review, we summarize the contribution of $\mathrm{K} 2 \mathrm{P}$ channels to neuronal excitability and to potassium homeostasis in glia. We describe recently discovered functions of K2P channels in glia, such as astrocytic passive conductance and glutamate release, microglial surveillance, and myelin generation by oligodendrocytes. We also discuss the potential role of glial K2P channels in neurological disorders. In the end, we discuss current limitations in K2P channel researches and suggest directions for future studies.
\end{abstract}

\section{Introduction}

Ion channels in the cell membrane, particularly potassium channels, play a vital role in the resting membrane potential of neurons and in the transmission of action potentials [1-5]. An appropriate resting membrane potential requires a leaky $\mathrm{K}^{+}$current, and two-pore-domain potassium (K2P) channels appear crucial in providing such current. These channels remain open ("leaky") across the range of physiological voltages, as well as during action potentials [6-8].

The first mammalian K2P channel, TWIK-1, was identified in humans in 1996 [9], and since then, another 14 members sharing a similar structure have been identified and organized into six subfamilies (TWIK, TREK, TASK, TALK, THIK, and TRESK) $[7,8]$. In contrast to other $\mathrm{K}^{+}$channels, which feature one pore domain per subunit, K2P channels contain two pore domains per subunit. TWIKs give rise to a weakly inwardly rectifying $\mathrm{K}^{+}$current, while TREKs are sensitive to mechanical and thermal stimuli as well as lipids.
TASKs and TALKs respond strongly to changes in extra- or intracellular $\mathrm{pH}$, while THIKs are sensitive to halothane.

$\mathrm{K} 2 \mathrm{P}$ channels are widely expressed in the nervous system, including the dorsal root ganglion (DRG), trigeminal ganglion (TG), spinal cord, cerebrum, and cerebellum $[10,11]$. In the peripheral nervous system, K2P channels are expressed in sensory neurons of the DRG and TG. In the brain, TWIK-1, TREK-1, TREK-2, TRAAK, TASK-1, and TASK-3 are preferentially expressed in specific regions. $\mathrm{K} 2 \mathrm{P}$ channels are also expressed in various patterns in different cell types in other brain regions.

Perhaps the best-understood functions of K2P channels are passive $\mathrm{K}^{+}$conductance and maintenance of resting membrane potential. Activation of K2P channels leads to the extrusion of $\mathrm{K}^{+}$into the extracellular space, hyperpolarizing the neuronal membrane and dampening neuronal excitability. Various endogenous and exogenous stimuli can regulate the activity of these ion channels, including membrane stretching, voltage, temperature, extra- and 
intracellular $\mathrm{pH}$, phospholipids, and other intracellular signaling molecules generated through the activation of $G$ protein-coupled receptors (GPCRs) [12].

Some K2P members are expressed not only in neurons but also in glial cells. Glia are nonneuronal cells in the nervous system that do not produce electrical impulses. Instead, they maintain homeostasis of certain ions, particularly $\mathrm{K}^{+}$, in the extracellular environment. In the brain, TASK-1 has been detected in astrocytes and oligodendrocytes, while TWIK-1 and TREK-1 are expressed in astrocytes, and THIK-1 was identified in microglia [13].

This review summarizes recent studies of K2P channel functions in neurons and glia of the central and peripheral nervous systems. These studies have relied primarily on genetic and pharmacological tools to elucidate channel function. We focus on how K2P channels in neurons help maintain resting membrane potential and regulate excitability, and on how $\mathrm{K} 2 \mathrm{P}$ channels in glia help maintain potassium homeostasis.

\section{K2P Channels and the Excitability of Neurons}

2.1. Peripheral Nervous System (PNS). Some K2P channels mediate nociception and pathological pain, and knocking them out in mice increases sensitivity to noxious stimuli $[14,15]$. These findings indicate a strong association between $\mathrm{K} 2 \mathrm{P}$ channels and neuronal excitability. Indeed, animal models of pathological pain show downregulation of K2P channels in the DRG or TG, as well as increased excitability of nociceptive neurons [16] (Figure 1).

2.1.1. TWIK-Related Spinal Cord $K^{+}$Channels (TRESKs). TRESK was first found in the human spinal cord, then discovered in mouse cerebrum [17]. In DRG neurons, this channel contributes strongly to background $\mathrm{K}^{+}$currents: mutating Gly339 to Arg in TRESK in mice significantly reduced standing outward current in DRG neurons, without affecting resting membrane potential $[18,19]$. At the same time, the mutation increased excitability of DRG neurons: it reduced the rheobase to elicit an action potential, and it increased the rate of action potential firing [18, 20, 21]. Knocking out TRESK in mice sensitized the animals to mechanical stress and cold, based on assessments of inflammatory and neuropathic pain responses, without substantially affecting their sensitivity to heat $[20,21]$. Bone metastasis in mice has been linked to depolarization of nociceptive neurons and to pain hypersensitivity, which were partially reversed by overexpressing TRESK in DRG neurons and exacerbated by knocking down TRESK [22]. Similarly, treating isolated DRG neurons with the TRESK inhibitor isobutylalkenyl amide depolarized the cells and evoked action potential [23].

TRESK has been linked to migraine pathophysiology, and a frameshift mutation (F139WfsX24) has been identified in patients suffering from migraine with aura [24]. In mice, this frameshift mutation showed a dominant-negative effect of reducing the rheobase and increasing the rate of action potential firing in TG, without altering resting membrane potential [25]. The frameshift may cause these effects by giving rise to a truncated protein, TRESK-MT2 [26]. Expression of TRESKMT2 in TG neurons of mice inhibited TREK-1 and TREK-2, reduced the rheobase, and increased the firing rate, indicating greater excitability. Using CRISPR-Cas9 to correct the frameshift in induced pluripotent stem cells from migraine patients reversed the hyperexcitability of the resulting differentiated nociceptors. Activating TRESK using cloxyquin in a mouse model of migraine alleviated pain hypersensitivity to mechanical and thermal stimuli [27]. Future studies are needed to examine coexpression of TRESK and TREK, to determine whether endogenous levels of TRESK-MT2 in human nociceptors inhibit TREK-1/2, and to evaluate the contribution of TREK-1/2 to migraine pathogenesis.

TRESK is also expressed in the nodose ganglia (NG), and NG neurons in diabetic rats show hyperpolarization as well as upregulated TRESK relative to NG neurons in healthy controls. [28, 29]. Silencing TRESK in diabetic animals partially restored normal excitability of NG neurons and thereby the animal's sensitivity to satiety signals [28, 29].

The fact that TRESK is expressed primarily in sensory neurons makes it a potential target for treating allodynia and migraine. Developing specific activators of this channel may expand on the range of analgesic agents currently available.

2.1.2. TREKs (TWIK-Related $\mathrm{K}^{+}$Channels). TREKs belong to another subfamily of K2P channels comprising TREK-1, TREK-2, and TRAAK. TREKs are sensitive to membrane stretching, $\mathrm{pH}$, arachidonic acid, and temperature, making them polymodal pain perception channels. In the DRG, TREKs are expressed in small-diameter neurons and to a small extent in medium- and large-diameter neurons [30]. Knocking out TREK-1, TREK-2, or TRAAK from mice rendered them more sensitive to thermal and mechanical stimuli [31-33], which indicated enhanced neuronal excitability. Downregulating TREK-2 in C-fiber nociceptors depolarized the membrane by about $10 \mathrm{mV}$ and increased spontaneous firing frequency. Downregulating TREK-2 also increased spontaneous pain behavior in a mouse model of complete Freund's adjuvant (CFA) [34].

Activating TREKs using the neuroprotective agent riluzole hyperpolarized the resting membrane in the superior cervical ganglion $[35,36]$. Similarly, activating TREK-1/2 in DRG neurons with BL-1249 or GI-530159 hyperpolarized the resting membrane and reduced firing frequency [37]. These results suggest that TREK activation in the peripheral nervous system can alleviate polymodal pathological pain, but the selectivity of those TREK activators has not been rigorously established. Potentially more reliable support for the therapeutic potential of TREK activation comes from studies with the recently developed TREK-1/2 activator C3001a [38]. C3001a was found to activate TREK-1 with a half-maximal effective concentration $\left(\mathrm{EC}_{50}\right)$ of $12.81 \mu \mathrm{M}$, and it activated TREK-2 with similar efficacy, while it did not appreciably activate TRAAK or other human K2P channels (TASK-3, TASK-1, TRESK, and THIK-1). Applying c3001a to dissociated DRG neurons produced weak hyperpolarization, increased the rheobase, and reduced the number of action potentials in small-sized neurons [38]. Applying the compound to mice with spared nerve injury or complete Freund's adjuvant alleviated spontaneous pain and cold hyperalgesia. The compound also alleviated mechanical allodynia and 


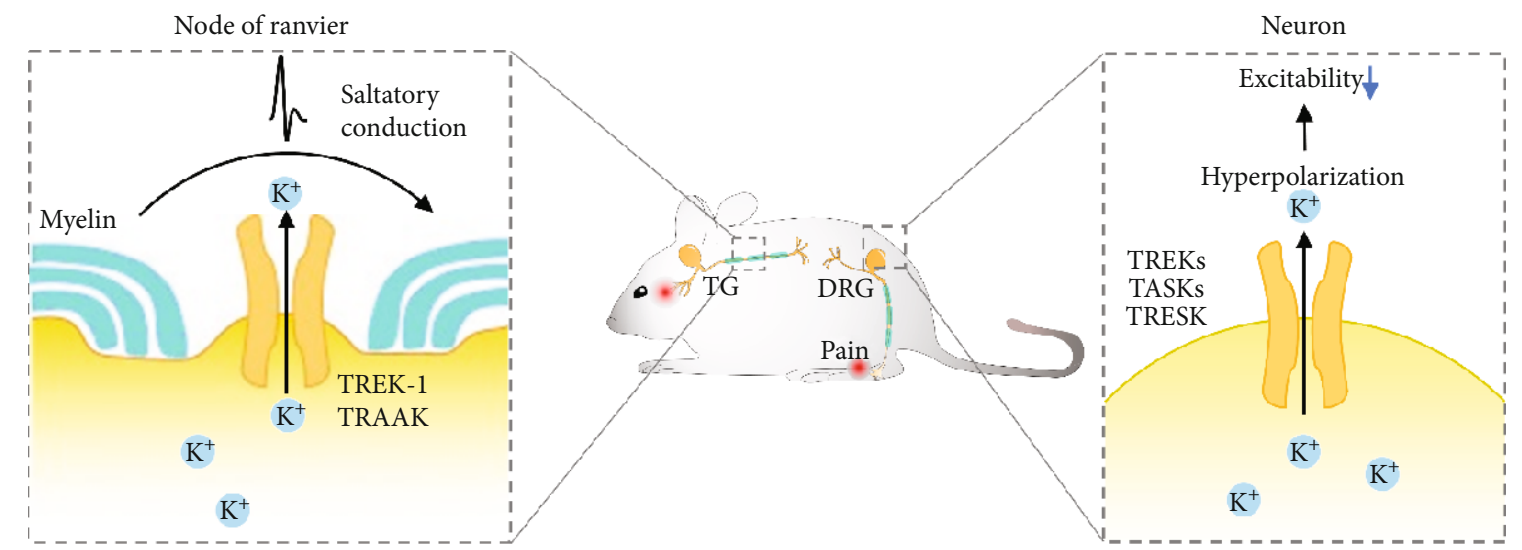

FIGURE 1: Functions of K2P channels in the peripheral nervous system. Left: TREK-1 and TRAAK are enriched in nodes of Ranvier on myelinated afferent nerves. The channels rapidly regenerate action potentials, allowing fast action potential conduction along the nerve. Right: K2P channels are expressed in neurons of the dorsal root ganglion and trigeminal ganglion. They extrude $\mathrm{K}^{+}$and hyperpolarize the membrane of neurons at rest, decreasing their excitability.

inflammation in a mouse model of pancreatitis. Thus, activating TREKs may be a useful therapeutic strategy.

TREK- 1 and TRAAK are enriched at nodes of Ranvier on myelinated afferent nerves. These nodes display unusual action potentials and quite leaky $\mathrm{K}^{+}$conductance that is thermo- and mechanosensitive. The nodes of Ranvier on nearly all trigeminal $\mathrm{A} \beta$-afferent nerves express both TREK-1 and TRAAK [39]. Knocking down or pharmacologically inhibiting either channel widened action potentials at the nodes, increased nodal membrane input resistance, and depolarized the resting membrane. Thus, these channels are probably required for rapid action potential repolarization at the nodes of Ranvier. The channels also permit rapid, high-frequency action potentials along myelinated afferent nerves. Consistent with these ideas, knocking down TREK1 or TRAAK in TG of rat significantly reduced tactile responses, suggesting sensory behavioral deficit [39]. These channels may play similar roles in other parts of the mammalian nervous system that contain rapidly conducting somatosensory afferent nerves or motor nerves. Further study is needed to determine whether other K2P channels are also involved in rapid action potential conduction at nodes of Ranvier in myelinated nerves in the peripheral and central nervous systems.

\subsubsection{TWIK-Related Acid-Sensitive $K^{+}$Channels (TASKs).} TASK-1 and TASK-3 belong to a subfamily of K2P channels sensitive to changes in extracellular $\mathrm{pH}[40]$, and they are expressed in DRG [11, 41]. TASK-3 is primarily expressed in small-sized DRG neurons, where it colocalizes with TRMP8, TRPV1, and tyrosine hydroxylase, suggesting selective expression in nociceptive neurons [42]. In the peripheral nervous system, TASK-3 is expressed in TRPM8-positive neurons, and knocking it out from mice rendered them hypersensitive to cold, and this effect was associated with depolarization of the resting membrane and increased amplitude of action potentials in TRPM8-positive neurons [42].

More details about the in vivo function of TASK-3 under physiological and pathological conditions began to emerge with the structure-based design of a selective TASK-3 activator, CHET3 [43]. Administering CHET3 to animal models of acute and chronic pain alleviated spontaneous pain as well as hyperalgesia in response to cold, heat, and mechanical stress. Applying CHET3 to isolated DRG neurons increased the rheobase and decreased action potential frequency, without altering resting membrane potential [43]. These genetic and pharmacological studies reveal a role for TASK-3 in membrane excitability of nociceptive neurons.

Since TASK-3 is expressed at much higher levels in the TG than DRG [43], future research should evaluate the potential role of TASK-3 in trigeminal diseases and facial sensation.

2.2. Central Nervous System. In the central nervous system, mRNAs encoding K2P channels have been detected in the cerebrum, cerebellum, brainstem, and spinal cord $[10,11]$. Neuronal K2P channels have been implicated in chronic pain [44], ischemia, epilepsy [45], sleep disorder, and major depressive disorder [46] (Figure 2).

2.2.1. TRESK. High expression of TRESK has been found in several areas of the central nervous system, including the cortex, periaqueductal gray (PAG), and dorsal horn of the spinal cord [47]. Spinal nerve ligation in mice to induce neuropathic pain is associated with TRESK upregulation in the superficial dorsal horn [48], and overexpressing TRESK in such animals alleviated their hyperalgesia, inflammation, and neuronal apoptosis $[49,50]$. These results suggest that TRESK in the spinal cord plays an essential role in pain perception, which needs to be confirmed in electrophysiological studies.

Recent evidence suggests that TRESK helps regulate nocturnal dynamics of the suprachiasmatic nucleus (SCN) and light-adaptive responses [51]. The SCN is the key circadian pacemaker, which synchronizes internal circadian rhythms to the external day-night cycle. TRESK expression increases in the early evening and remains high throughout the night, suggesting a rhythmic expression pattern. In the animal study, neurons at rest in the SCN of control animals were 


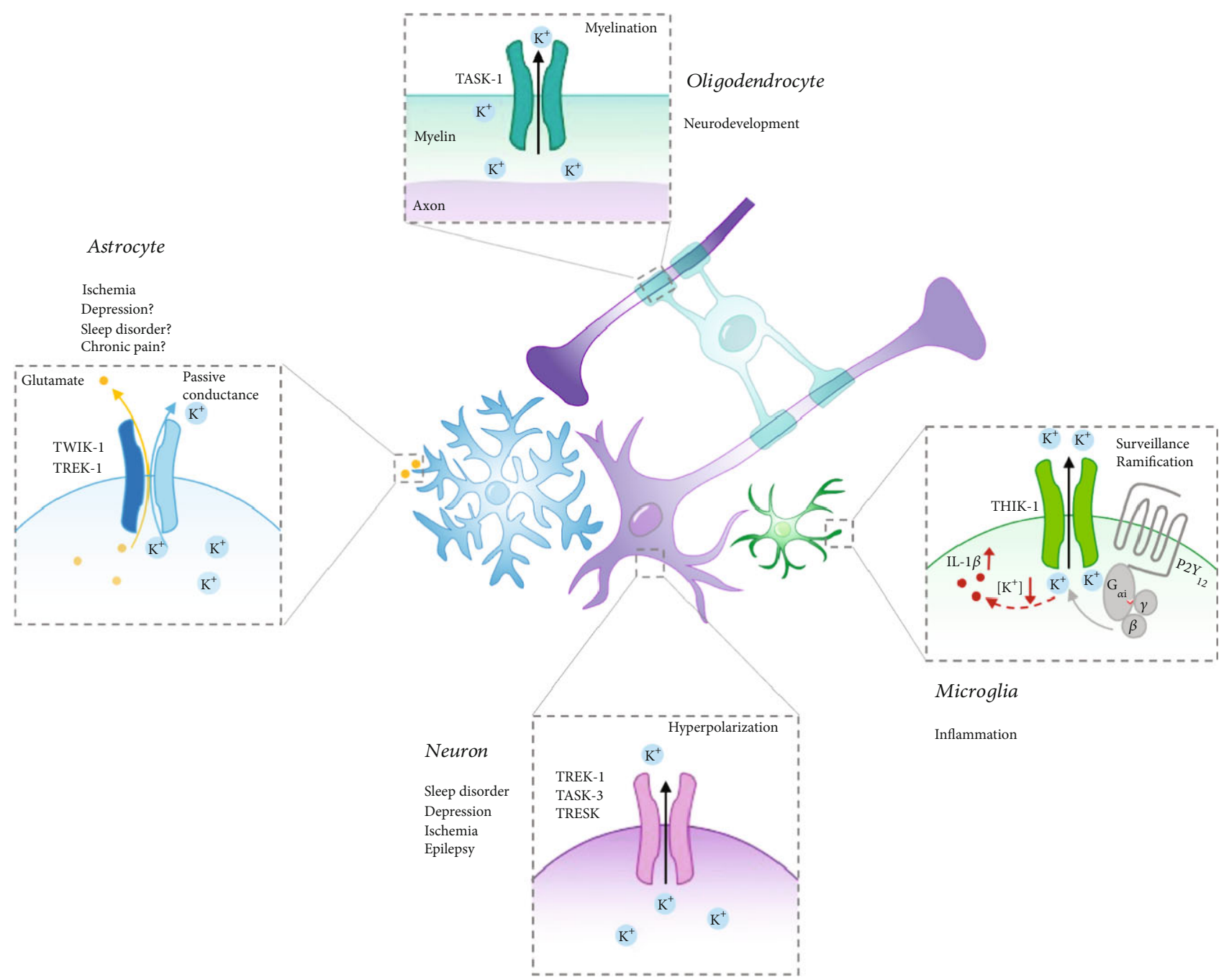

Figure 2: Roles of K2P channels in the central nervous system. K2P channels regulate neuronal excitability and contribute to many normal and disease processes, including sleep, epilepsy, ischemia, and depression. In glia, K2P channels maintain glial membrane potential and are involved in astrocytic passive conductance and glutamate release, microglial surveillance, myelin generation by oligodendrocytes, and $\mathrm{K}^{+}$ homeostasis.

more depolarized during the day than at night, whereas the same neurons in animals lacking TRESK remained constantly in a depolarized state, and their nocturnal spike rate was higher than in controls. In addition, knocking out TRESK led to a much weaker $\mathrm{Ca}^{2+}$ response to glutamate [51].

2.2.2. TREKs. TREK-1 and TRAAK are widely expressed across the central nervous system, with TREK-1 most abundant in the striatum, cortex and hippocampus. TRAAK is most abundant in the cortex. TREK-2 is restricted to the cerebellar granule cell layer $[11,52,53]$.

(1) TREK-1. TREK-1 can regulate the excitability of neurons, but its precise roles may depend on the type of neuron. In pyramidal neurons of the CA1 region of the hippocampus, TREK-1 deficiency depolarized resting neurons, reduced the rheobase, and increased action potential frequency [54]. Similarly, in serotonergic neurons of the dorsal raphe nucleus
(DRN), TREK-1 knockout increased discharge frequency in mice [55]. The channel does not, however, appear to affect resting membrane potential of GABAergic neurons in the striatum [56]. Treating brain slices with the endogenous TREK-1 inhibitor spadin or the inhibitor SID1900 increased the firing rate of serotonergic neurons in the DRN $[57,58]$. Spadin also depolarized cortical neurons at rest [59]. The various effects of TREK-1 have been implicated in nervous system disorders as described below:

(1) Depression: knocking out TREK-1 from several mouse models of depression alleviated depression symptoms, as measured in the forced swimming test, tail suspension test, conditioned suppression of motility test, and learned helplessness test [55]. In fact, the knockout animals showed similar behavior as wild-type mice treated with the antidepressants fluoxetine or paroxetine. Knockout was associated 
with higher firing rates of serotonergic neurons, which likely increased the release of serotonin into target structures. Mice lacking TREK-1 were insensitive to selective serotonin reuptake inhibitors, suggesting that the efficacy of such inhibitors involves TREK-1 inhibition [55]. Indeed, these inhibitors appear to inhibit TREK-1 in a dose-dependent manner $[55,60]$. Both spadin and SID1900 enhanced the excitability of serotonergic neurons in mice, alleviating depressive symptoms $[57,58]$. TREK-1 knockdown in hippocampal neurons alleviated depressive symptoms in a mouse model of lipopolysaccharideinduced depression [61]. Consistent with the studies on rodent models, four single nucleotide polymorphisms in TREK-1 were also identified in some patients with treatment resistance in major depressive disorders [62]

These observations suggest that TREK-1 may be a useful therapeutic target in depression. Future study is needed to determine whether TREK-1 actively regulates serotonin release and is involved in the mechanism of action of selective serotonin reuptake inhibitors.

(2) Ischemia and seizure: knocking out TREK-1 from mice increased their risk of ischemia due to transient bilateral occlusion of the common carotid arteries or due to occlusion of the aortic arch and left subclavian artery [56]. This ischemia was associated with higher mortality. Knockout also increased risk of epileptic seizures triggered by kainite or pentylenetetrazol; the mutant animals showed increased morbidity as well as increased spike amplitude and frequency, based on electroencephalography [56]. Administering spadin to mice did not reduce infarct size following focal ischemia or alleviate kainate-induced seizures [63]

TREK-1 in serotonergic neurons of the dorsal raphe has recently been implicated in the circadian photoperiod [64]. Longer photoperiods may reduce TREK-1 function, since pharmacological inhibition of TREK-1 significantly increased spike frequency in animals exposed to short or equinox photoperiods but not in animals exposed to long photoperiods, which expressed lower levels of the channel [64].

(2) TREK-2/TRAAK. TREK-2 is a downstream mediator of $\mathrm{GABA}_{\mathrm{B}}$ receptors in neurons of the entorhinal cortex, giving the channel a strong influence over spatial learning. Treating the stellate neuron of the entorhinal cortex with the $\mathrm{GABA}_{\mathrm{B}}$ receptor activator baclofen activated TREK-2, which blocked action potentials and hyperpolarized the membrane through mechanisms involving the $G_{a i}, G_{a o}$, and PKA pathways [65]. This decrease in neuronal excitability led to impaired spatial learning, which was abolished when TREK-2 was knocked down. Knockdown did not alter the resting membrane potential of stellate neurons in the entorhinal cortex.
In another study, norepinephrine was shown to activate TREK-2 and thereby hyperpolarize neurons at rest in the superficial layers of the entorhinal cortex. This mechanism appeared to involve $\alpha_{-}{ }_{2 \mathrm{~A}}$ adrenergic receptors as well as the $\mathrm{G}_{\alpha \mathrm{i}}$ and PKA pathways [66].

In humans, FHEIG (facial dysmorphism, hypertrichosis, epilepsy, intellectual disability/developmental delay, and gingival overgrowth) was associated with a missense mutation in TRAAK. The recombinant mutant TRAAK channel showed a significant gain of function basally and damaged sensitivity to mechanical stimulus and arachidonic acid [67].

2.2.3. TASKs. TASKs are widely expressed across the central nervous system $[10,68]$. TASK-1 may be most abundant in the cerebellum, while TASK-3 may be more abundant in the hippocampus, cortex, cerebellum, and certain nuclei, such as the paraventricular nuclei of the thalamus, locus coeruleus, and the dorsal raphe [11]. TASK electrophysiology has been studied in cerebellar granule neurons of rats and serotonergic raphe neurons of mice $[69,70]$. Knocking out TASK-1 from mice did not alter resting membrane potential of cerebellar granule neurons or the properties of their action potentials [71]. In contrast, knocking out TASK-3 depolarized cerebellar granule neurons by $10 \mathrm{mV}$ and decreased both the rheobase and action potential amplitude [72]. These results suggest that TASK-3 may contribute more to the resting membrane potential of cerebellar granule neurons than TASK-1.

In mature neurons, transmembrane chloride gradients are mainly regulated by KCC2 and NKCC1, two cationchloride cotransporters, which, respectively, mediate outward and inward cotransport of $\mathrm{Cl}^{-}$and $\mathrm{K}^{+}$under physiological conditions. Consistent with this idea, chronic knockdown of KCC2 in rat hippocampus increased neuronal excitability by downregulating levels of TASK-3 at the membrane [73]. Altogether, these studies indicate that TASKs stabilize neuronal membrane potential and regulate their activity. As a result, these channels likely play roles in epilepsy, sleep disorder, and depression, as described below.

(1) Epilepsy. In the rat entorhinal cortex, application of serotonin depolarized GABAergic interneurons at rest, increased action potential firing frequency, and increased GABA release [74]. As a result, pyramidal cells showed lower excitability. These effects of serotonin were associated with inhibition of TASK-3 in interneurons, suggesting that the channel may be a target in treating epilepsy. Future research is required to determine whether selective inhibition of TASK-3 can alleviate one or more subtypes of epilepsy.

The Kcnk9 gene coding for TASK-3 is located at chromosomal position $8 \mathrm{q} 24$, a locus associated with absence epilepsy $[75,76]$, although the association is somewhat controversial [77]. A mutation in TASK-3 has been linked to absence epilepsy in a rat model, but mutant and wild-type animals showed similar leaky $\mathrm{K}^{+}$currents [78]. Further work is needed to clarify whether and how this mutation contributes to absence seizures. 
(2) Sleep Disorder. Sleep is essential to emotional health, but the molecular processes that determine daily sleep duration and the sleep-wake cycle remain elusive. Thalamocortical neuronal networks alternate between burst activity during sleep and tonic single-spike activity during wakefulness [79]. Inhibition of TASK-1 and TASK-3 depolarized thalamic relay neurons, leading them to engage preferentially in tonic single-spike activity $[80,81]$. Similarly, inhibiting TASK-3 using spermine during repetitive activity or hypoxia depolarized the thalamic neurons by about $8 \mathrm{mV}$ and switched the firing mode from burst to tonic [82]. Thus, TASKs have potential roles in the sleep-wake cycle.

Consistent with this idea, animals expressing TASK-3 mutants showed increased nocturnal activity and shorter REM sleep [83, 84]. Animals deficient in TASK-3 lacked an $\theta$ oscillation that was seen in the cortical electroencephalogram of control animals and that resembled type II $\theta$. The deficient animals also progressed much more slowly from wakefulness to sleep, and their sleep episodes and REM $\theta$ oscillations were more fragmented [79]. Knocking out TASK-3 severely shortened sleep [85]. Exposing mice to chronic sleep fragmentation depolarized medial habenula cholinergic output neurons at rest and increased the frequency of their spontaneous firing. Inhibition of TASK-3 mimicked the sleep fragmentation effects in control mice, while the effects of TASK-3 inhibition were absent in sleep fragmentation mice [86]. These results suggest that TASK-3 mediates the ability of sleep fragmentation to increase the excitability of medial habenula cholinergic output neurons.

These findings identify TASK-3 as a mediator of sleep disturbance, but how TASK-3 regulates firing patterns is still unclear. Further study is needed to investigate how TASK-3 contributes to the normal sleep cycle and how its impairment contributes to emotional disorders related to sleep disturbance, such as depression and anxiety.

(3) Depression. Knocking out TASK-3 from mice impaired working memory, based on the T-maze spontaneous alternation test, and it damaged spatial memory, based on the Morris water maze test [83]. At the same time, TASK-3 knockout alleviated depressive behaviors, as assessed in the forced swimming test and tail suspension test [84]. These observations identify TASK-3 as a potential target in the treatment of depression. In fact, the receptor may help mediate the antidepressive effects of cannabinoid receptor agonists in mouse models [87-89]: knocking out TASK-3 rendered the animals less responsive to the agonist WIN55212-2 mesylate [83]. Future research is needed to validate how TASK-3 regulates depression.

Dysfunction of TASK-3 was also identified to cause neurological disorders in humans. A missense mutation in TASK-3 was identified to abolish the channel's currents and resulted in a maternally transmitted genomicimprinting syndrome characterized by mental retardation, hypotonia, and dimorphisms in patients [90,91].
2.2.4. Tandem of Pore Domains in a Weak Inward Rectifying $K^{+}$Channel (TWIK). TWIK-1 appears to be expressed widely across the central nervous system [92], but few studies have examined its physiological roles in neurons. In the entorhinal cortex, serotonin activated TWIK-1, which hyperpolarized stellate and pyramidal neurons at rest in superficial layers, and it slowed their action potential firing [93]. It is unclear whether TWIK-1 in the entorhinal cortex acts as a homodimer or as a heterodimer with another K2P channel isotype.

In dentate gyrus granule cells, downregulating TWIK-1 depolarized the cells at rest and increased their firing rate [94]. TASK-3 appeared to localize in the proximal dendrites and soma of those cells, similar to the localization of TWIK-1. Knocking down both TWIK-1 and TASK-3 depolarized the cells at rest and increased their action potential firing rate [95]. These results suggest that TWIK-1 and TASK-3 contribute to the intrinsic excitability of dentate gyrus granule cells.

\section{K2P and the Potassium Homeostasis of Glia}

Glia support neuronal functions via various mechanisms in the central nervous system, and $\mathrm{K} 2 \mathrm{P}$ channels are expressed in glia. A transcriptome analysis detected the mRNAs encoding TWIK-1 and TREK-1 in astrocytes [96], and immunohistochemistry detected TASK-1 protein in astrocytes of hippocampus and oligodendrocytes in the mouse brain [97]. Several studies have examined the functions of K2P channels in glia (Figure 2), though much more work remains to be done.

3.1. Astrocytes. Astrocytes are star-shaped, specialized glia that differentiate from a neural stem cell pool. They primarily mediate ionic homeostasis and provide structural support to neurons. They also contribute to neurotransmitter release and synaptic development. Astrocyte dysfunction, termed reactive astrogliosis, is common in injury or disease affecting the central nervous system, including some neurological diseases related to aging: Huntington's disease, Alzheimer's disease (AD), and Parkinson's disease [98].

3.1.1. Astrocyte and Passive Conductance. TREK-1 and TWIK-1 in astrocytes contribute to the large $\mathrm{K}^{+}$conductance of cultured cortical astrocytes [13]. While knocking out TWIK-1 from mice did not significantly alter astrocytic passive conductance [99], depleting it from cultured astrocytes led to more negative resting membrane potential [100]. In contrast, eliminating TREK-1 from cultured astrocytes did not alter either resting membrane potential or passive potassium conductance [99]. On the other hand, treating cultured astrocytes or hippocampal slices with spadin reduced their passive conductance by inhibiting the activity of TWIK1/TREK-1 heterodimers [101]. These data suggest that TREK-1 mildly contributes to the passive conductance of astrocytes. Knocking out TREK-1 alone or together with TWIK-1 did not alter the expression of other potassium channels, suggesting that the other channels do not compensate for K2P channel activity [102]. 
3.1.2. Glutamate Release. Glutamate is one of the most important neurotransmitters that are released from excitatory presynaptic neurons and that mediate neuronal transmission. Emerging evidence suggests that astrocytes can also release glutamate to modulate synaptic plasticity, neuronal excitability, and transmitter release in physiological and pathophysiological states [103-105]. How astrocytes release glutamate is controversial: for example, does it depend on channels, transporters, or vesicular exocytosis? Several studies in cultured astrocytes and brain slices suggest that increased intracellular $\mathrm{Ca}^{2+}$ concentration induces glutamate release via a pathway involving the exocytotic machinery [106]. However, these findings may not be entirely reliable, given that the supporting experiments relied on nonspecific methods to manipulate intracellular $\mathrm{Ca}^{2+}$ concentration and glutamate transport in astrocytes. For example, the rise of intracellular $\mathrm{Ca}^{2+}$ also activates the $\mathrm{Ca}^{2+}$-activated $\mathrm{K}^{+}$ channels in astrocytes and extrudes $\mathrm{K}^{+}$onto surrounding neurons, depolarizing them and probably resulting in glutamate release [106]. Using a metabotropic glutamate receptor agonist to induce a change in $\mathrm{Ca}^{2+}$ concentration in astrocytes may also trigger other changes due to activation of the same receptor on neurons. Indeed, pharmacological inhibition of certain anion channels can reduce glutamate release from astrocytes independently of the exocytotic machinery [103].

Brief synaptic activity can trigger $\mathrm{K}^{+}$uptake via $\mathrm{K} 2 \mathrm{P}$ channels containing TREK-1, leading to a temporary increase in astrocyte volume. The volume returns to normal when bestrophin-1 allows $\mathrm{Cl}^{-}$out of the cell [107]. TREK-1 is responsible for fast glutamate release from astrocytes, and the $\mathrm{Ca}^{2+}$-activated $\mathrm{Cl}^{-}$channel bestrophin-1 is responsible for slow glutamate release [108]. Fast release depends on activation of $\mathrm{G}_{\alpha \mathrm{i}}$ and dissociation of $\mathrm{G}_{\beta \gamma}$, followed by interaction between $\mathrm{G}_{\beta \gamma}$ and TREK-1, leading to the opening of glutamate-permeable TREK-1 [109]. This overall pathway is mediated by $\mathrm{G}_{\mathrm{i}}$-type $\mathrm{G}$ protein-coupled receptors including cannabinoid receptor 1 , adenosine receptor $\mathrm{A} 1$, and $\mu$ opioid receptor. Like TREK-1, TWIK-1 can directly interact with the $\mathrm{G}_{\gamma}$ subunit; in the presence of $\mathrm{G}_{\gamma}$, the heterodimer TWIK-1/TREK-1, which is expressed in astrocytes $[13,96$, $100,110]$, becomes permeable to glutamate and $\mathrm{K}^{+}[100]$. These data suggest that $G_{\beta \gamma}$ alters the pore region of the K2P channel. Further studies are needed to determine how TWIK-1/TREK-1 heterodimers are permeable to glutamate.

3.1.3. Ischemia. Astrocytic TREK-1 is also involved in the pathology of ischemia. In an animal model of focal ischemia induced by arterial occlusion, reperfusion was associated with upregulation of TREK-1 [111]. Inhibition of TREK-1 impaired astrocytic clearance and exacerbated inflammation after ischemia, resulting in neuronal apoptosis [112]. During ischemia, the ischemic metabolite lactate upregulates TREK1 in astrocytes via the PKA pathway [113]. Astrocyte elevated gene-1 (AEG-1) also induces TREK-1 expression in astrocytes during ischemia [114].

These observations suggest that K2P channels, particularly TREK-1, may be involved in passive $\mathrm{K}^{+}$conductance, glutamate release, and ischemia pathology. Future work, such as using conditional knockout mice, should focus on establishing which K2P channels are expressed in astrocytes and what are their functions.

3.2. Microglia. Microglia fulfill various functions within the central nervous system, which are related mainly to immune responses and homeostasis. Microglia monitor for damage, injury, or disease, and they contribute to synaptic pruning. Disruption of microglial function leads to several diseases, and microglia-mediated neuroinflammation, associated with synaptic loss and cognitive decline [115], is a characteristic of late $\mathrm{AD}$. Dysregulation of synaptic pruning is associated with cognitive defects in autism [116].

Little is known regarding the expression and function of K2P channels in microglia. THIK-1 appears to play a critical role in microglial ramification and immune surveillance in the brain [117]. Microglia in rat brain slices showed a more depolarized potential $(-40.6 \mathrm{mV})$ and higher input resistance than neurons or other glia. Locally puffing $100 \mathrm{mM}$ ATP onto microglia activated the $\mathrm{P}_{2} \mathrm{Y}_{12}$ receptor and hyperpolarized their membrane by $\sim 30 \mathrm{mV}$ [117]. Pharmacological blockade or genetic ablation of THIK-1 depolarized the microglia at rest, inhibiting microglial ramification and surveillance. These results suggest that by modulating resting membrane potential of microglia, THIK-1 may help regulate microglia function. Blocking THIK-1 in brain slices with quinine, bupivacaine, or tetrapentylammonium abolished the release of interleukin- $1 \beta$ from microglia [117]. This implies that THIK-1 may be involved in the pathological function of microglia in brain diseases. Future studies should investigate the link between resting membrane potential and microglial function, especially since the resting membrane potential of microglia varies across brain regions. Future research should also clarify the region and cell type specificity of THIK-1 expression in the brain.

3.3. Oligodendrocytes. The primary function of oligodendrocytes is to insulate the neuronal axon by creating a myelin sheath, which contributes to rapid signal transduction. TASK-1 can induce currents in oligodendrocytes in response to extracellular acidification, and lack of TASK-1 depolarizes oligodendroglial precursor cells [97]. Inhibition of TASK-1 can protect oligodendrocytes from ischemic injury [118]. TASK-1 may be involved in multiple sclerosis, but the details are unclear. In multiple sclerosis, insufficient recruitment and differentiation of oligodendroglial precursor cells leads to incomplete remyelination. The demyelination and inflammation in the central nervous system of the mice model of multiple sclerosis was alleviated by treatment with bupivacaine, which acts against TASK-1 as well as other channels [97], but not by specific TASK-1 knockout or inhibition $[119,120]$. In another mouse model of multiple sclerosis, TASK-1 knockout increased the number of mature oligodendrocytes and accelerated developmental myelination, yet it did not affect oligodendroglial differentiation during remyelination after pathological demyelination [97]. Future studies should clarify whether and how TASK-containing K2P channels help mediate oligodendroglial differentiation and remyelination in normal and disease contexts. 


\section{Future Perspectives}

$\mathrm{K} 2 \mathrm{P}$ channels, which differ from $\mathrm{K}_{\mathrm{v}}$ and $\mathrm{K}_{\mathrm{ir}}$ channels, produce leaky $\mathrm{K}^{+}$currents to regulate neuronal excitability. $\mathrm{K} 2 \mathrm{P}$ channels are distributed widely in the peripheral and central nervous systems, where their expression patterns vary. In neurons, $\mathrm{K} 2 \mathrm{P}$ channels modulate resting membrane potential and action potentials, allowing them to influence multiple biological functions. In the peripheral nervous system, TRESK, TASK, and TREK are involved in nociception and pathological pain. Activation of these channels reduces neuronal excitability and thereby alleviates allodynia in several animal models of pain, suggesting that these channels might be potent targets in pain management. In the central nervous system, activation of $\mathrm{G}$ protein-coupled receptors induces $\mathrm{K} 2 \mathrm{P}$ channels to regulate neuronal excitability in ways that can influence chronic pain, ischemia, epilepsy, sleep disorder, and major depressive disorder.

While neuronal K2P channels regulate excitability, glial $\mathrm{K} 2 \mathrm{P}$ channels are likely more involved in $\mathrm{K}^{+}$and neurotransmitter homeostasis. TREK-1, TWIK-1, THIK-1, and TASK-1 are thought to be expressed in glia, but further investigation is needed to confirm the regional and cell-type distribution of $\mathrm{K} 2 \mathrm{P}$ channels. Indeed, much more work is needed to elucidate the functions of K2P channels in glia as well as in neurons, such as through animal studies in which channels are knocked out of specific cell types under physiological and pathological conditions.

\section{Conflicts of Interest}

The authors have no conflict of interest regarding this article.

\section{Acknowledgments}

This study was supported by grants to R.J. from the National Natural Science Foundation of China (32071003), the Department of Science and Technology of Sichuan Province (2020ZYD006), and the 1.3.5 Project for Disciplines of Excellence of West China Hospital, Sichuan University (ZYJC21034).

\section{References}

[1] D. J. Adams, S. J. Smith, and S. H. Thompson, "Ionic currents in molluscan soma," Annual Review of Neuroscience, vol. 3, no. 1, pp. 141-167, 1980.

[2] A. M. Katz, F. C. Messineo, and L. Herbette, "Ion channels in membranes," Circulation, vol. 65, 1, Part 2, pp. I2-10, 1982.

[3] O. M. Sejersted and G. Sjogaard, "Dynamics and consequences of potassium shifts in skeletal muscle and heart during exercise," Physiological Reviews, vol. 80, no. 4, pp. 1411$1481,2000$.

[4] L. Y. Jan and Y. N. Jan, "Voltage-gated potassium channels and the diversity of electrical signalling," Journal of Physiology, vol. 590, no. 11, pp. 2591-2599, 2012.

[5] N. Schmitt, M. Grunnet, and S. P. Olesen, "Cardiac potassium channel subtypes: new roles in repolarization and arrhythmia," Physiological Reviews, vol. 94, no. 2, pp. 609653, 2014.
[6] F. Lesage and M. Lazdunski, "Molecular and functional properties of two-pore-domain potassium channels," American Journal of Physiology: Renal Physiology, vol. 279, no. 5, pp. F793-F801, 2000.

[7] F. V. Sepulveda, L. Pablo Cid, J. Teulon, and M. I. Niemeyer, "Molecular aspects of structure, gating, and physiology of $\mathrm{pH}$-sensitive background K2P and Kir K+-transport channels," Physiological Reviews, vol. 95, no. 1, pp. 179-217, 2015.

[8] P. Enyedi and G. Czirjak, "Molecular background of leak K+ currents: two-pore domain potassium channels," Physiological Reviews, vol. 90, no. 2, pp. 559-605, 2010.

[9] F. Lesage, E. Guillemare, M. Fink et al., "TWIK-1, a ubiquitous human weakly inward rectifying $\mathrm{K}+$ channel with a novel structure," EMBO Journal, vol. 15, no. 5, pp. 10041011, 1996.

[10] A. D. Medhurst, G. Rennie, C. G. Chapman et al., "Distribution analysis of human two pore domain potassium channels in tissues of the central nervous system and periphery," Molecular Brain Research, vol. 86, no. 1-2, pp. 101-114, 2001.

[11] E. M. Talley, G. Solorzano, Q. Lei, D. Kim, and D. A. Bayliss, "CNS distribution of members of the two-pore-domain (KCNK) potassium channel family," Journal of Neuroscience, vol. 21, no. 19, pp. 7491-7505, 2001.

[12] N. Decher, S. Rinne, M. Bedoya, W. Gonzalez, and A. K. Kiper, "Molecular pharmacology of $\mathrm{K}_{2 \mathrm{P}}$ potassium channels," Cell Physiol Biochem, vol. 55, no. S3, pp. 87-107, 2021.

[13] M. Zhou, G. Xu, M. Xie et al., "TWIK-1 and TREK-1 are potassium channels contributing significantly to astrocyte passive conductance in rat hippocampal slices," Journal of Neuroscience, vol. 29, no. 26, pp. 8551-8564, 2009.

[14] C. Tsantoulas, "Emerging potassium channel targets for the treatment of pain," Current Opinion in Supportive \& Palliative Care, vol. 9, no. 2, pp. 147-154, 2015.

[15] A. Mathie and E. L. Veale, "Two-pore domain potassium channels: potential therapeutic targets for the treatment of pain," Pflügers Archiv - European Journal of Physiology, vol. 467, no. 5, pp. 931-943, 2015.

[16] X. Y. Li and H. Toyoda, "Role of leak potassium channels in pain signaling," Brain Research Bulletin, vol. 119, Part A, pp. 73-79, 2015.

[17] Y. Sano, K. Inamura, A. Miyake et al., "A Novel Two-pore Domain $\mathrm{K}^{+}$Channel, TRESK, Is Localized in the Spinal Cord," Journal of Biological Chemistry, vol. 278, no. 30, pp. 27406-27412, 2003.

[18] T. Dobler, A. Springauf, S. Tovornik et al., "TRESK two-poredomain $\mathrm{K}^{+}$channels constitute a significant component of background potassium currents in murine dorsal root ganglion neurones," Journal of Physiology, vol. 585, no. 3, pp. 867-879, 2007.

[19] D. Kang and D. Kim, "TREK-2 (K2P10.1) and TRESK (K2P18.1) are major background $\mathrm{K}^{+}$channels in dorsal root ganglion neurons," American Journal of Physiology-Cell Physiology, vol. 291, no. 1, pp. C138-C146, 2006.

[20] A. Castellanos, A. Pujol-Coma, A. Andres-Bilbe et al., "TRESK background $\mathrm{K}^{+}$channel deletion selectively uncovers enhanced mechanical and cold sensitivity," Journal of Physiology, vol. 598, no. 5, pp. 1017-1038, 2020.

[21] Z. Guo, C. S. Qiu, X. Jiang et al., "TRESK K ${ }^{+}$Channel activity regulates trigeminal nociception and headache," eNeuro, vol. 6 , no. 4, 2019. 
[22] Y. Yang, S. Li, Z. R. Jin et al., "Decreased abundance of TRESK two-pore domain potassium channels in sensory neurons underlies the pain associated with bone metastasis," Science Signaling, vol. 11, no. 552, article eaao5150, 2018.

[23] A. Tulleuda, B. Cokic, G. Callejo, B. Saiani, J. Serra, and X. Gasull, "TRESK channel contribution to nociceptive sensory neurons excitability: modulation by nerve injury," Molecular Pain, vol. 7, p. 1744-8069-7-30, 2011.

[24] R. G. Lafrenière, M. Z. Cader, J. F. Poulin et al., "A dominantnegative mutation in the TRESK potassium channel is linked to familial migraine with aura," Nature Medicine, vol. 16, no. 10, pp. 1157-1160, 2010.

[25] P. Liu, Z. Xiao, F. Ren et al., "Functional analysis of a migraine-associated TRESK $\mathrm{K}^{+}$channel mutation," Journal of Neuroscience, vol. 33, no. 31, pp. 12810-12824, 2013.

[26] P. Royal, A. Andres-Bilbe, P. Ávalos Prado et al., "Migraineassociated TRESK mutations increase neuronal excitability through alternative translation initiation and inhibition of TREK," Neuron, vol. 101, no. 2, pp. 232-245.e6, 2019.

[27] P. Pettingill, G. A. Weir, T. Wei et al., "A causal role for TRESK loss of function in migraine mechanisms," Brain, vol. 142, no. 12, pp. 3852-3867, 2019.

[28] G. Grabauskas, X. Wu, S. Zhou, J. Y. Li, J. Gao, and C. Owyang, "High-fat diet-induced vagal afferent dysfunction via upregulation of 2-pore domain potassium TRESK channel," JCI Insight, vol. 4, no. 17, 2019.

[29] G. Grabauskas, X. Wu, I. Song, S. Y. Zhou, T. Lanigan, and C. Owyang, "Increased Activation of the TRESK K ${ }^{+}$Mediates Vago-Vagal Reflex Malfunction in Diabetic Rats," Gastroenterology, vol. 151, no. 5, pp. 910-922.e7, 2016.

[30] V. Viatchenko-Karpinski, J. Ling, and J. G. Gu, "Characterization of temperature-sensitive leak $\mathrm{K}^{+}$currents and expression of TRAAK, TREK-1, and TREK2 channels in dorsal root ganglion neurons of rats," Molecular Brain, vol. 11, no. 1, p. 40, 2018.

[31] A. Alloui, K. Zimmermann, J. Mamet et al., "TREK-1, a $\mathrm{K}^{+}$ channel involved in polymodal pain perception," $E M B O$ Journal, vol. 25, no. 11, pp. 2368-2376, 2006.

[32] V. Pereira, J. Busserolles, M. Christin et al., "Role of the TREK2 potassium channel in cold and warm thermosensation and in pain perception," Pain, vol. 155, no. 12, pp. 2534-2544, 2014.

[33] J. Noël, K. Zimmermann, J. Busserolles et al., "The mechanoactivated $\mathrm{K}^{+}$channels TRAAK and TREK-1 control both warm and cold perception," EMBO Journal, vol. 28, no. 9, pp. 1308-1318, 2009.

[34] C. Acosta, L. Djouhri, R. Watkins, C. Berry, K. Bromage, and S. N. Lawson, "TREK2 expressed selectively in IB4-binding C-fiber nociceptors hyperpolarizes their membrane potentials and limits spontaneous pain," Journal of Neuroscience, vol. 34, no. 4, pp. 1494-1509, 2014.

[35] P. Rivas-Ramirez, A. Cadaveira-Mosquera, J. A. Lamas, and A. Reboreda, "Muscarinic modulation of TREK currents in mouse sympathetic superior cervical ganglion neurons," European Journal of Neuroscience, vol. 42, no. 2, pp. 17971807, 2015.

[36] D. Fernández-Fernández, A. Cadaveira-Mosquera, L. RuedaRuzafa et al., "Activation of TREK currents by riluzole in three subgroups of cultured mouse nodose ganglion neurons," PLoS One, vol. 13, no. 6, article e0199282, 2018.
[37] A. J. C. Loucif, P. P. Saintot, J. Liu et al., "GI-530159, a novel, selective, mechanosensitive two-pore-domain potassium (K2P) channel opener, reduces rat dorsal root ganglion neuron excitability," British Journal of Pharmacology, vol. 175, no. 12, pp. 2272-2283, 2018.

[38] Y. Qiu, L. Huang, J. Fu et al., “TREK channel family activator with a well-defined structure-activation relationship for pain and neurogenic inflammation," Journal of Medicinal Chemistry, vol. 63, no. 7, pp. 3665-3677, 2020.

[39] H. Kanda, J. Ling, S. Tonomura, K. Noguchi, S. Matalon, and J. G. Gu, "TREK-1 and TRAAK Are Principal $\mathrm{K}^{+}$Channels at the Nodes of Ranvier for Rapid Action Potential Conduction on Mammalian Myelinated Afferent Nerves," Neuron, vol. 104, no. 5, pp. 960-971.e7, 2019.

[40] Y. Kim, H. Bang, and D. Kim, "TASK-3, a New Member of the Tandem Pore $\mathrm{K}^{+}$Channel Family," Journal of Biological Chemistry, vol. 275, no. 13, pp. 9340-9347, 2000.

[41] B. Marsh, C. Acosta, L. Djouhri, and S. N. Lawson, "Leak K channel mRNAs in dorsal root ganglia: Relation to inflammation and spontaneous pain behaviour," Molecular and Cellular Neurosciences, vol. 49, no. 3, pp. 375-386, 2012.

[42] C. Morenilla-Palao, E. Luis, C. Fernández-Peña et al., "Ion channel profile of TRPM8 cold receptors reveals a role of TASK-3 potassium channels in thermosensation," Cell Reports, vol. 8, no. 5, pp. 1571-1582, 2014.

[43] P. Liao, Y. Qiu, Y. Mo et al., "Selective activation of TWIKrelated acid-sensitive $\mathrm{K}^{+} 3$ subunit-containing channels is analgesic in rodent models," Science Translational Medicine, vol. 11, no. 519, article eaaw8434, 2019.

[44] G. García, R. Noriega-Navarro, V. A. Martínez-Rojas, E. J. Gutiérrez-Lara, N. Oviedo, and J. Murbartián, "Spinal TASK-1 and TASK-3 modulate inflammatory and neuropathic pain," European Journal of Pharmacology, vol. 862, p. 172631, 2019.

[45] R. Kohling and J. Wolfart, "Potassium channels in epilepsy," Cold Spring Harbor Perspectives in Medicine, vol. 6, no. 5, 2016.

[46] M. Borsotto, J. Veyssiere, H. Moha ou Maati, C. Devader, J. Mazella, and C. Heurteaux, "Targeting two-pore domain $\mathrm{K}^{+}$channels TREK-1 and TASK-3 for the treatment of depression: a new therapeutic concept," British Journal of Pharmacology, vol. 172, no. 3, pp. 771-784, 2015.

[47] S. Yoo, J. Liu, M. Sabbadini, P. Au, G. X. Xie, and C. S. Yost, "Regional expression of the anesthetic-activated potassium channel TRESK in the rat nervous system," Neuroscience Letters, vol. 465, no. 1, pp. 79-84, 2009.

[48] H. Y. Hwang, E. Zhang, S. Park et al., "TWIK-related spinal cord $\mathrm{K}^{+}$Channel expression is increased in the spinal dorsal horn after spinal nerve ligation," Yonsei Medical Journal, vol. 56, no. 5, pp. 1307-1315, 2015.

[49] J. Zhou, W. Lin, H. Chen, Y. Fan, and C. Yang, "TRESK contributes to pain threshold changes by mediating apoptosis via MAPK pathway in the spinal cord," Neuroscience, vol. 339, pp. 622-633, 2016.

[50] G. T. Kim, A. S. Siregar, E. J. Kim et al., "Upregulation of TRESK channels contributes to motor and sensory recovery after spinal cord injury," International Journal of Molecular Sciences, vol. 21, no. 23, p. 8997, 2020.

[51] T. Lalic, A. Steponenaite, L. Wei et al., "TRESK is a key regulator of nocturnal suprachiasmatic nucleus dynamics and 
light adaptive responses," Nature Communications, vol. 11, no. 1 , p. $4614,2020$.

[52] G. J. Hervieu, J. E. Cluderay, C. W. Gray et al., "Distribution and expression of TREK-1, a two-pore-domain potassium channel, in the adult rat CNS," Neuroscience, vol. 103, no. 4, pp. 899-919, 2001.

[53] W. Gu, G. Schlichthörl, J. R. Hirsch et al., "Expression pattern and functional characteristics of two novel splice variants of the two-pore-domain potassium channel TREK-2," Journal of Physiology, vol. 539, no. 3, pp. 657-668, 2002.

[54] W. Wang, C. M. Kiyoshi, Y. du et al., “TREK-1 null impairs neuronal excitability, synaptic plasticity, and cognitive function," Molecular Neurobiology, vol. 57, no. 3, pp. 13321346, 2020.

[55] C. Heurteaux, G. Lucas, N. Guy et al., "Deletion of the background potassium channel TREK-1 results in a depressionresistant phenotype," Nature Neuroscience, vol. 9, no. 9, pp. 1134-1141, 2006.

[56] C. Heurteaux, N. Guy, C. Laigle et al., "TREK-1, a K ${ }^{+}$channel involved in neuroprotection and general anesthesia," $E M B O$ Journal, vol. 23, no. 13, pp. 2684-2695, 2004.

[57] J. Mazella, O. Pétrault, G. Lucas et al., "Spadin, a sortilinderived peptide, targeting rodent TREK-1 channels: a new concept in the antidepressant drug design," PLoS Biology, vol. 8, no. 4, article e1000355, 2010.

[58] D. Ye, Y. Li, X. Zhang et al., "TREK1 channel blockade induces an antidepressant-like response synergizing with 5$\mathrm{HT}_{1 \mathrm{~A}}$ receptor signaling," European Neuropsychopharmacology, vol. 25, no. 12, pp. 2426-2436, 2015.

[59] C. Devader, A. Khayachi, J. Veyssière et al., "In vitro and in vivo regulation of synaptogenesis by the novel antidepressant spadin," British Journal of Pharmacology, vol. 172, no. 10, pp. 2604-2617, 2015.

[60] L. E. Kennard, J. R. Chumbley, K. M. Ranatunga, S. J. Armstrong, E. L. Veale, and A. Mathie, "Inhibition of the human two-pore domain potassium channel, TREK-1, by fluoxetine and its metabolite norfluoxetine," British Journal of Pharmacology, vol. 144, no. 6, pp. 821-829, 2005.

[61] A. Kim, H. G. Jung, Y. E. Kim et al., "The knockdown of TREK-1 in hippocampal neurons attenuate lipopolysaccharide-induced depressive-like behavior in mice," International Journal of Molecular Sciences, vol. 20, no. 23, p. 5902, 2019.

[62] R. H. Perlis, P. Moorjani, J. Fagerness et al., "Pharmacogenetic analysis of genes implicated in rodent models of antidepressant response: association of TREK1 and treatment resistance in the STAR*D study," Neuropsychopharmacology, vol. 33, no. 12, pp. 2810-2819, 2008.

[63] H. Moha ou Maati, J. Veyssiere, F. Labbal et al., "Spadin as a new antidepressant: absence of TREK-1-related side effects," Neuropharmacology, vol. 62, no. 1, pp. 278-288, 2012.

[64] M. A. Giannoni-Guzmán, A. Kamitakahara, V. Magalong, P. Levitt, and D. G. McMahon, "Circadian photoperiod alters TREK-1 channel function and expression in dorsal raphe serotonergic neurons via melatonin receptor 1 signaling," Journal of Pineal Research, vol. 70, no. 2, article e12705, 2021.

[65] P. Y. Deng, Z. Xiao, C. Yang et al., "GABA ${ }_{B}$ Receptor Activation Inhibits Neuronal Excitability and Spatial Learning in the Entorhinal Cortex by Activating TREK- $2 \mathrm{~K}^{+}$Channels," Neuron, vol. 63, no. 2, pp. 230-243, 2009.
[66] Z. Xiao, P. Y. Deng, L. Rojanathammanee et al., "Noradrenergic Depression of Neuronal Excitability in the Entorhinal Cortex via Activation of TREK-2 $\mathrm{K}^{+}$Channels," Journal of Biological Chemistry, vol. 284, no. 16, pp. 10980-10991, 2009.

[67] C. K. Bauer, P. Calligari, F. C. Radio et al., "Mutations in KCNK4 that affect gating cause a recognizable neurodevelopmental syndrome," American Journal of Human Genetics, vol. 103, no. 4, pp. 621-630, 2018.

[68] Z. Rusznák, K. Pocsai, I. Kovács et al., "Differential distribution of TASK-1, TASK-2 and TASK-3 immunoreactivities in the rat and human cerebellum," Cellular and Molecular Life Sciences, vol. 61, no. 12, pp. 1532-1542, 2004.

[69] J. Han, J. Truell, C. Gnatenco, and D. Kim, "Characterization of four types of background potassium channels in rat cerebellar granule neurons," Journal of Physiology, vol. 542, no. 2, pp. 431-444, 2002.

[70] C. P. Washburn, J. E. Sirois, E. M. Talley, P. G. Guyenet, and D. A. Bayliss, "Serotonergic raphe neurons express TASK channel transcripts and a TASK-like $\mathrm{pH}$ - and halothanesensitive $\mathrm{K}^{+}$conductance," Journal of Neuroscience, vol. 22, no. 4, pp. 1256-1265, 2002.

[71] M. I. Aller, E. L. Veale, A. M. Linden et al., "Modifying the subunit composition of TASK channels alters the modulation of a leak conductance in cerebellar granule neurons," Journal of Neuroscience, vol. 25, no. 49, pp. 11455-11467, 2005.

[72] S. G. Brickley, M. I. Aller, C. Sandu et al., "TASK-3 two-pore domain potassium channels enable sustained high-frequency firing in cerebellar granule neurons," Journal of Neuroscience, vol. 27, no. 35, pp. 9329-9340, 2007.

[73] M. Goutierre, S. al Awabdh, F. Donneger et al., "KCC2 regulates neuronal excitability and hippocampal activity via interaction with TASK-3 channels," Cell Reports, vol. 28, no. 1, pp. 91-103.e7, 2019.

[74] P. Y. Deng and S. Lei, "Serotonin increases GABA release in rat entorhinal cortex by inhibiting interneuron TASK-3 $\mathrm{K}^{+}$ channels," Molecular and Cellular Neurosciences, vol. 39, no. 2, pp. 273-284, 2008.

[75] F. A. de Falco, P. Striano, A. de Falco et al., "Benign adult familial myoclonic epilepsy: genetic heterogeneity and allelism with ADCME," Neurology, vol. 60, no. 8, pp. 13811385, 2003.

[76] Y. Sugimoto, R. Morita, K. Amano et al., "Childhood absence epilepsy in 8q24: refinement of candidate region and construction of physical map," Genomics, vol. 68, no. 3, pp. 264-272, 2000.

[77] C. Kananura, T. Sander, S. Rajan et al., “Tandem pore domain $\mathrm{K}^{+}$-channel TASK-3 (KCNK9) and idiopathic absence epilepsies," American Journal of Medical Genetics, vol. 114, no. 2, pp. 227-229, 2002.

[78] J. Holter, D. Carter, N. Leresche, V. Crunelli, and P. Vincent, "A TASK3 channel (KCNK9) mutation in a genetic model of absence epilepsy," Journal of Molecular Neuroscience, vol. 25, no. 1, pp. 037-052, 2005.

[79] T. C. Gent, M. Bandarabadi, C. G. Herrera, and A. R. Adamantidis, "Thalamic dual control of sleep and wakefulness," Nature Neuroscience, vol. 21, no. 7, pp. 974-984, 2018.

[80] S. G. Meuth, T. Budde, T. Kanyshkova, T. Broicher, T. Munsch, and H. C. Pape, "Contribution of TWIK-related acid-sensitive $\mathrm{K}^{+}$channel 1 (TASK1) and TASK3 channels to the control of activity modes in thalamocortical neurons," Journal of Neuroscience, vol. 23, no. 16, pp. 6460-6469, 2003. 
[81] S. G. Meuth, T. Kanyshkova, P. Meuth et al., "Membrane resting potential of thalamocortical relay neurons is shaped by the interaction among TASK3 and HCN2 channels," Journal of Neurophysiology, vol. 96, no. 3, pp. 1517-1529, 2006.

[82] B. Musset, S. G. Meuth, G. X. Liu et al., "Effects of divalent cations and spermine on the $\mathrm{K}^{+}$channel TASK-3 and on the outward current in thalamic neurons," Journal of Physiology, vol. 572, no. 3, pp. 639-657, 2006.

[83] A. M. Linden, C. Sandu, M. I. Aller et al., "TASK-3 knockout mice exhibit exaggerated nocturnal activity, impairments in cognitive functions, and reduced sensitivity to inhalation anesthetics," Journal of Pharmacology and Experimental Therapeutics, vol. 323, no. 3, pp. 924-934, 2007.

[84] A. L. Gotter, V. P. Santarelli, S. M. Doran et al., "TASK-3 as a potential antidepressant target," Brain Research, vol. 1416, pp. 69-79, 2011.

[85] D. S. Pang, C. J. Robledo, D. R. Carr et al., "An unexpected role for TASK-3 potassium channels in network oscillations with implications for sleep mechanisms and anesthetic action," Proceedings of the National Academy of Sciences of the United States of America, vol. 106, no. 41, pp. 1754617551, 2009.

[86] F. Ge, P. Mu, R. Guo et al., "Chronic sleep fragmentation enhances habenula cholinergic neural activity," Molecular Psychiatry, vol. 26, no. 3, pp. 941-954, 2021.

[87] G. Gobbi and P. Blier, "Effect of neurokinin-1 receptor antagonists on serotoninergic, noradrenergic and hippocampal neurons: comparison with antidepressant drugs," Peptides, vol. 26, no. 8, pp. 1383-1393, 2005.

[88] W. Jiang, Y. Zhang, L. Xiao et al., "Cannabinoids promote embryonic and adult hippocampus neurogenesis and produce anxiolytic- and antidepressant-like effects," Journal of Clinical Investigation, vol. 115, no. 11, pp. 3104-3116, 2005.

[89] Z. Zhang, W. Wang, P. Zhong et al., "Blockade of 2arachidonoylglycerol hydrolysis produces antidepressantlike effects and enhances adult hippocampal neurogenesis and synaptic plasticity," Hippocampus, vol. 25, no. 1, pp. 16-26, 2015.

[90] J. M. Graham Jr., N. Zadeh, M. Kelley et al., "KCNK9 imprinting syndrome-further delineation of a possible treatable disorder," American Journal of Medical Genetics. Part A, vol. 170, no. 10, pp. 2632-2637, 2016.

[91] O. Barel, S. A. Shalev, R. Ofir et al., "Maternally Inherited Birk Barel Mental Retardation Dysmorphism Syndrome Caused by a Mutation in the Genomically Imprinted Potassium Channel_KCNK9_," American Journal of Human Genetics, vol. 83, no. 2, pp. 193-199, 2008.

[92] F. Lesage, I. Lauritzen, F. Duprat et al., "The structure, function and distribution of the mouse TWIK-1 $\mathrm{K}^{+}$channel," FEBS Letters, vol. 402, no. 1, pp. 28-32, 1997.

[93] P. Y. Deng, S. K. Poudel, L. Rojanathammanee, J. E. Porter, and S. Lei, "Serotonin inhibits neuronal excitability by activating two-pore domain $\mathrm{K}+$ channels in the entorhinal cortex," Molecular Pharmacology, vol. 72, no. 1, pp. 208-218, 2007.

[94] O. Yarishkin, D. Y. Lee, E. Kim et al., "TWIK-1 contributes to the intrinsic excitability of dentate granule cells in mouse hippocampus," Molecular Brain, vol. 7, no. 1, p. 80, 2014.

[95] J. H. Choi, O. Yarishkin, E. Kim et al., "TWIK-1/TASK-3 heterodimeric channels contribute to the neurotensin-mediated excitation of hippocampal dentate gyrus granule cells,"
Experimental and Molecular Medicine, vol. 50, no. 11, pp. 1-13, 2018.

[96] J. D. Cahoy, B. Emery, A. Kaushal et al., “A transcriptome database for astrocytes, neurons, and oligodendrocytes: a new resource for understanding brain development and function," Journal of Neuroscience, vol. 28, no. 1, pp. 264-278, 2008.

[97] S. Albrecht, S. Korr, L. Nowack et al., "The K2P-channel TASK1 affects oligodendroglial differentiation but not myelin restoration," Glia, vol. 67, no. 5, pp. 870-883, 2019.

[98] S. Liddelow and B. Barres, "SnapShot: astrocytes in health and disease," Cell, vol. 162, no. 5, pp. 1170-1170.e1, 2015.

[99] W. Wang, A. Putra, G. P. Schools et al., "The contribution of TWIK-1 channels to astrocyte $\mathrm{K}^{+}$current is limited by retention in intracellular compartments," Frontiers in Cellular Neuroscience, vol. 7, p. 246, 2013.

[100] E. Mi Hwang, E. Kim, O. Yarishkin et al., "A disulphidelinked heterodimer of TWIK-1 and TREK-1 mediates passive conductance in astrocytes," Nature Communications, vol. 5, no. 1, p. 3227, 2014.

[101] Y. Bae, J. H. Choi, K. Ryoo et al., "Spadin modulates astrocytic passive conductance via inhibition of TWIK-1/TREK-1 heterodimeric channels," International Journal of Molecular Sciences, vol. 21, no. 24, p. 9639, 2020.

[102] Y. du, C. M. Kiyoshi, Q. Wang et al., "Genetic deletion of TREK-1 or TWIK-1/TREK-1 potassium channels does not alter the basic electrophysiological properties of mature hippocampal astrocytes in situ," Frontiers in Cellular Neuroscience, vol. 10, p. 13, 2016.

[103] A. Verkhratsky and M. Nedergaard, "Physiology of astroglia," Physiological Reviews, vol. 98, no. 1, pp. 239-389, 2018.

[104] M. D. Scofield, "Exploring the role of astroglial glutamate release and association with synapses in neuronal function and behavior," Biological Psychiatry, vol. 84, no. 11, pp. 778-786, 2018.

[105] T. A. Fiacco, C. Agulhon, and K. D. McCarthy, "Sorting out astrocyte physiology from pharmacology," Annual Review of Pharmacology and Toxicology, vol. 49, pp. 151-174, 2009.

[106] N. B. Hamilton and D. Attwell, "Do astrocytes really exocytose neurotransmitters?," Nature Reviews Neuroscience, vol. 11, no. 4, pp. 227-238, 2010.

[107] J. Woo, M. W. Jang, J. Lee, W. Koh, K. Mikoshiba, and C. J. Lee, "The molecular mechanism of synaptic activityinduced astrocytic volume transient," Journal of Physiology, vol. 598, no. 20, pp. 4555-4572, 2020.

[108] D. H. Woo, K. S. Han, J. W. Shim et al., "TREK-1 and Best1 channels mediate fast and slow glutamate release in astrocytes upon GPCR activation," Cell, vol. 151, no. 1, pp. 2540, 2012.

[109] D. H. Woo, J. Y. Bae, M. H. Nam et al., "Activation of astrocytic $\mu$-opioid receptor elicits fast glutamate release through TREK-1-containing K2P channel in hippocampal astrocytes," Frontiers in Cellular Neuroscience, vol. 12, p. 319, 2018.

[110] J. Benesova, V. Rusnakova, P. Honsa et al., "Distinct expression/function of potassium and chloride channels contributes to the diverse volume regulation in cortical astrocytes of GFAP/EGFP mice," PLoS One, vol. 7, no. 1, article e29725, 2012.

[111] M. Wang, J. Song, W. Xiao et al., "Changes in lipid-sensitive two-pore domain potassium channel TREK-1 expression and 
its involvement in astrogliosis following cerebral ischemia in rats," Journal of Molecular Neuroscience, vol. 46, no. 2, pp. 384-392, 2012.

[112] X. Wu, Y. Liu, X. Chen et al., "Involvement of TREK-1 activity in astrocyte function and neuroprotection under simulated ischemia conditions," Journal of Molecular Neuroscience, vol. 49, no. 3, pp. 499-506, 2013.

[113] A. Banerjee, S. Ghatak, and S. K. Sikdar, "l-Lactate mediates neuroprotection against ischaemia by increasing TREK1 channel expression in rat hippocampal astrocytes in vitro," Journal of Neurochemistry, vol. 138, no. 2, pp. 265-281, 2016.

[114] A. Kim, H. G. Jung, S. C. Kim et al., "Astrocytic AEG-1 regulates expression of TREK-1 under acute hypoxia," Cell Biochem Funct, vol. 38, no. 2, pp. 167-175, 2020.

[115] S. Hong, V. F. Beja-Glasser, B. M. Nfonoyim et al., "Complement and microglia mediate early synapse loss in Alzheimer mouse models," Science, vol. 352, no. 6286, pp. 712-716, 2016.

[116] I. Voineagu, X. Wang, P. Johnston et al., "Transcriptomic analysis of autistic brain reveals convergent molecular pathology," Nature, vol. 474, no. 7351, pp. 380-384, 2011.

[117] C. Madry, V. Kyrargyri, I. L. Arancibia-Cárcamo et al., "Microglial Ramification, Surveillance, and Interleukin-1 $\beta$ Release Are Regulated by the Two-Pore Domain $\mathrm{K}^{+}$Channel THIK-1," Neuron, vol. 97, no. 2, pp. 299-312.e6, 2018.

[118] V. Hawkins and A. Butt, "TASK-1 channels in oligodendrocytes: a role in ischemia mediated disruption," Neurobiology of Disease, vol. 55, pp. 87-94, 2013.

[119] S. Bittner, M. A. Bauer, P. Ehling et al., "The TASK1 channel inhibitor A293 shows efficacy in a mouse model of multiple sclerosis," Experimental Neurology, vol. 238, no. 2, pp. 149$155,2012$.

[120] S. Bittner, S. G. Meuth, K. Göbel et al., "TASK1 modulates inflammation and neurodegeneration in autoimmune inflammation of the central nervous system," Brain, vol. 132, no. 9, pp. 2501-2516, 2009. 